\title{
The short-term effects of PMMA contact lens wear on keratometric behaviour: results for a single keratoconic cornea*
}

\section{E Chetty ${ }^{\dagger}$ and WDH Gillan}

\author{
${ }^{\dagger}$ Department of Optometry, University of Johannesburg, PO Box 524 Auckland Park, 2006 South \\ Africa \\ $\dagger<$ elizabethchetty2@gmail.com > \\ $\ddagger<$ wgillan@uj.ac.za $>$
}

Received 12 February 2010; revised version accepted 9 June 2010

\begin{abstract}
Keratometric behaviour is a multifaceted issue that many researchers have investigated for years. Many internal and external influences can have an effect on the cornea's keratometric behaviour. This investigation forms a small part of a larger study that aims at determining the effects that rigid contact lenses might have on keratometric behaviour. This pilot study examined the keratometric behaviour of a single, mildly keratoconic cornea that was fitted with a polymethyl methacrylate (PMMA) contact lens. Sixty successive auto-keratometric
\end{abstract}

measurements were taken immediately before and immediately after three hours of contact lens wear. The data obtained was transformed to dioptric power matrices and were analysed using multivariate statistical methods. This study showed that, at least in one keratoconic cornea, there appeared to be a statistically significant change in corneal curvature under the influence of a PMMA contact lens. The contact lens had also appeared to decrease variation in corneal curvature. There was no control study done on this eye therefore the effects of diurnal variation, if any, could not be established. ( $S$ Afr Optom 2010 69(2) 69-76)

\section{Introduction}

Keratometric behaviour can be described as the overall change in an individual's keratometric or anterior corneal state over time which is influenced by known and unknown factors, both of which cause continuous fluctuations in the corneal curvature ${ }^{1}$. The introduction of a contact lens onto the corneal surface is one of the known factors that affect keratometric behaviour but the full extent of its effect remains vague. Studies conducted in an attempt to understand the influence that a rigid contact lens has on keratometric behaviour have all concluded that there is generally an increase in corneal curvature ${ }^{2-6}$. However, the methods used for data analysis in these studies were either incomplete or incorrect.

A more recent study ${ }^{7}$, which made use of more appropriate multivariate methods of analysis, led to the discovery that apart from the expected overall stigmatic increase in corneal curvature there were also ortho-antistigmatic changes (an increase in corneal

$\dagger$ BOptom (UJ)

*DipOptom DPhil(RAU) CAS(NewEnCO) FAAO FIACLE

*This paper results from preliminary research towards the degree Magister Philosophiae (MPhil) in Optometry at the University of Johannesburg undertaken by E Chetty with the guidance of Professor WDH Gillan. 
curvature in the vertical meridian accompanied by a decrease in corneal curvature in the horizontal meridian). All of the above mentioned studies investigated normal healthy corneas only. The CLEK (Collaborative Longitudinal Evaluation of Keratoconus) study, ${ }^{8}$ has monitored corneal curvature changes in keratoconic patients (who were fitted with rigid gas permeable contact lenses) over an eight year period and has reported increased corneal curvatures in keratoconic corneas as well. However, in our opinion, this increase in corneal curvature reported could possibly be linked to disease progression and not necessarily to rigid contact lens wear.

Although poorly understood, keratoconus can be easily identified by the characteristic signs of the disease which include corneal thinning, Vogt's striae, Fleisher's ring, corneal scarring, scissoring reflex with retinoscopy, reduced spectacle compensated visual acuity and an oil drop reflex with ophthalmoscopy. Instruments such as topographers and keratometers also assist clinicians in making their diagnosis of keratoconus ${ }^{10}$. Mild keratoconus can usually be managed with spectacle correction or soft contact lenses, however, as the disease progresses, rigid contact lenses become necessary to provide satisfactory vision. As mentioned before, placing a contact lens onto the corneal surface will probably influence keratometric behaviour. The aim of this study was to investigate the short term effects of PMMA contact lens wear on keratometric behaviour in keratoconic patients. To our knowledge, there have not been any studies of this nature published to date. Auto-keratometric measurements were taken immediately before and immediately after three hours of PMMA contact lens wear in an attempt to understand how a non gaspermeable rigid contact lens affects keratometric behaviour. This study is also unprecedented in that the data obtained were analysed using multivariate statistical methods.

\section{Methods}

A 21-year-old male patient was referred to the University of Johannesburg's Optometry clinic for an eye examination for suspected keratoconus. This patient had been using spectacle compensation for five years and complained that his current spectacles no longer provided him with adequate vision for his daily tasks. The case history revealed that the patient did not have previous experience with contact lens wear. He did not have a family history of keratoconus nor did he have any health problems. Although there was no history of atopy, he did report eye rubbing. This patient fitted the criteria that we set for our pilot study, that is, he was a mild keratoconic (as classified by the Oculus Pentacam), had no previous history of contact lens wear and he was a healthy individual with no other systemic or ocular problems other than the keratoconus. This study involved taking auto-keratometric measurements immediately before and immediately after the tolerance trial (which took place from 10am to $1 \mathrm{pm}$ ). The tolerance trial is a clinically accepted trial period of three hours of PMMA contact lens wear that all new potential contact lens patients go through before dispensing regular use rigid gas permeable contact lenses. The trial period is long enough to be a provocative test in that any corneal compromise that may be induced by the presence of a contact lens will probably occur within the three hour period. During this consultation the patient was informed about the prospective study and gave his informed consent to continue as a study subject. The results of some of the preliminary tests are presented in Table 1 .

Table 1. Results from refraction, best compensated visual acuity and slitlamp biomicroscopy.

\begin{tabular}{|l|l|l|}
\hline & RIGHT EYE & LEFT EYE \\
\hline Spectacle refraction (\#7A) & $-0.25 /-5.50 \times 55$ & $-0.50 /-4.50 \times 130$ \\
\hline Compensated visual acuity & $6 / 9$ & $6 / 18$ \\
\hline $\begin{array}{l}\text { Slitlamp biomicroscopy } \\
\text { (specifically signs of } \\
\text { keratoconus) }\end{array}$ & $\begin{array}{l}\text { - slight corneal thinning } \\
- \text { Fleischer's ring } \\
\text { - increased nerve visibility } \\
\text { - no Vogt's striae } \\
\text { - no corneal scarring }\end{array}$ & $\begin{array}{l}\text { Similar findings to that of } \\
\text { the right eye. }\end{array}$ \\
\hline
\end{tabular}


It was evident from the preliminary testing that the left eye was more severely affected. Clinical experience has suggested that instruments such as the Pentacam do not necessarily trigger automatically and that the auto-keratometer cannot be focussed adequately if the keratoconic cornea is in advanced stages, therefore it was decided that only the right eye be used for this study to ensure that the instruments were adequately utilized.

On the second consultation the patient was fitted with various trial lenses in an attempt to find the best cornea-to-lens fitting relationship (both eyes were fitted but for the purpose of this study only the details of the right eye are deemed pertinent). The right eye was eventually fitted with a tricurve contact lens of base curve $7.4 \mathrm{~mm}$, optic zone diameter of $7.50 \mathrm{~mm}$, total diameter of $9.30 \mathrm{~mm}$ and power of $-3 \mathrm{D}$. The patient's visual acuity improved to $6 / 6$ with an over-refraction of -2 D. Corneal topography can be estimated with the aid of the Pentacam measurement and fluorescein fit as illustrated in Figures 1 and 2 respectively.

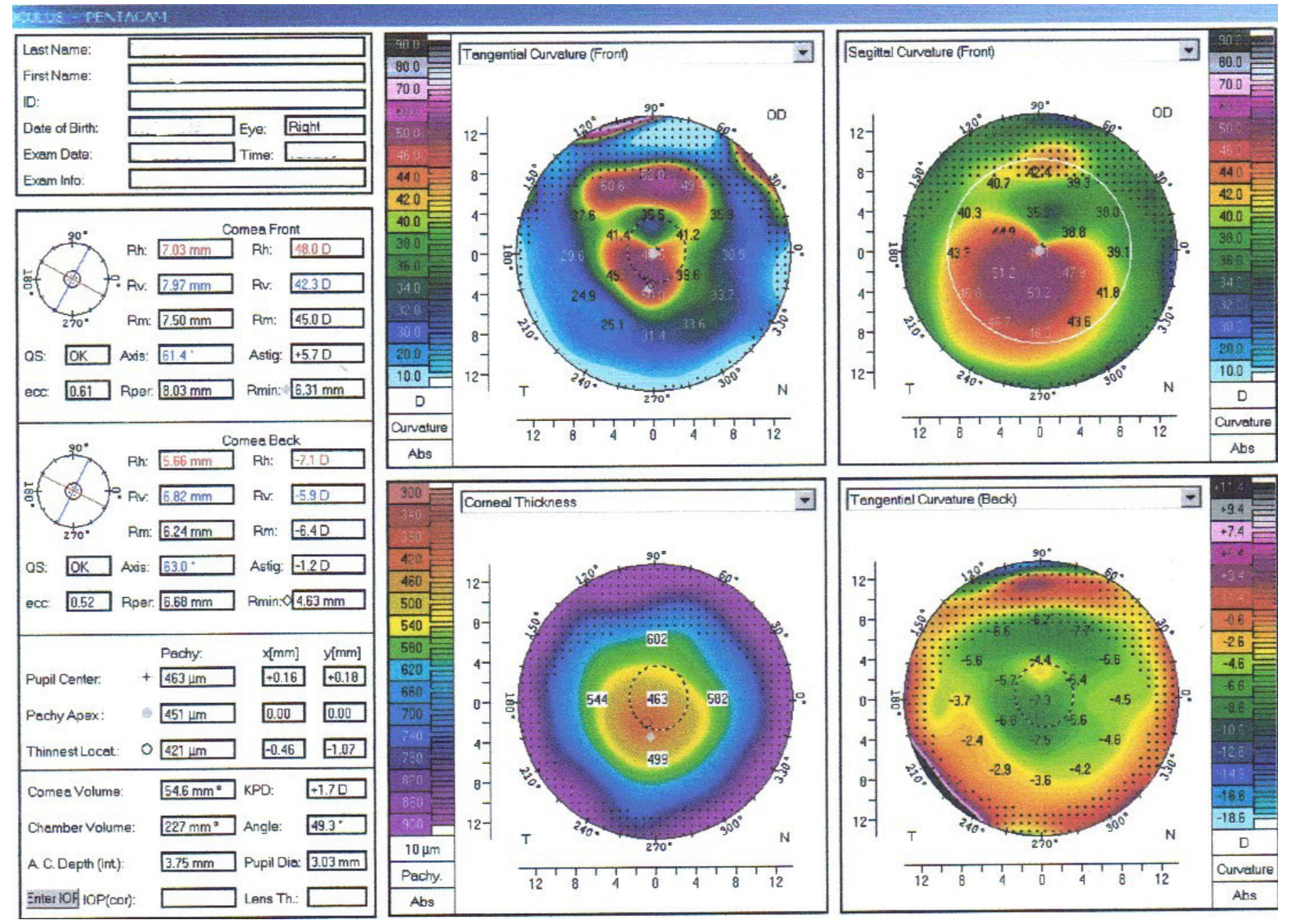

Figure 1. Results obtained from the Oculus Pentacam for the patient's right eye illustrating front tangential curvature (top left), front sagittal curvature (top right), corneal thickness (bottom left) and back tangential curvature (bottom right). The column on the left provides quantitive information about the dimensions of the cornea. 


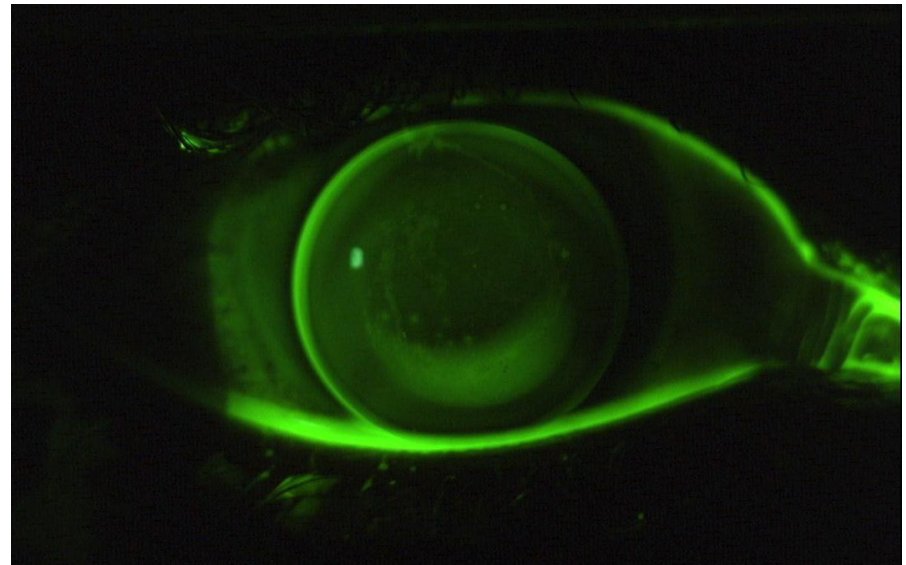

Figure 2. Slitlamp photograph of the fluorescein pattern of the $7.4 \mathrm{~mm}$ base curve PMMA contact lens on the keratoconic cornea of the patient's right eye. As is evident in the photograph, there is slight central corneal touch with incomplete mid-peripheral bearing and peripheral clearance.

Before the tolerance trial commenced during the third consultation, 60 auto-keratometric measurements were taken of the right eye with a Nidek ARK700 auto-keratometer. The auto-keratometer was the instrument of choice for this study as it provided a swift method of taking keratometric measurements. The auto-keratometer provides less variation and bias than the manual keratometer ${ }^{11}$ and the accuracy of this instrument has been established in previous investigations $^{12,13}$. The patient was seated in front of the auto-keratometer and instructed to keep both eyes open, blink normally and fixate on the instrument's target whilst measurements were taken. He was also asked to remove his head from the forehead and chin rest between measurements so that the instrument could be re-aligned for each measurement. It took approximately 29 minutes for the first set of 60 measurements. The pre-selected trial lenses were then inserted and he was asked to return after three hours. The patient was also asked not to do any strenuous physical activity as this has been shown to affect keratometric behaviour ${ }^{14}$. When the patient returned three hours later, his eyes were checked with a slitlamp for any signs of corneal compromise. The contact lenses were then removed and immediately another 60 autokeratometric measurements were taken of the right eye. The duration of the second set of measurements was 19 minutes. The keratometric data collected was transformed into dioptric power matrices. This was achieved using software designed specifically for this purpose by Harris and Malan ${ }^{15-18}$ with modifications made by Rubin. The transformed data was in a suitable form to allow for multivariate statistical methods of analysis.

\section{Results}

Stereo-pair scatter plots facilitate our understanding of statistical data by providing a visual representation of each measurement. One can observe the three dimensional percept of the stereo-pairs by allowing one's eyes to drift into an exo-position (outwards). Each dot on the scatter plot is a dioptric power representation of each keratometric measurement taken. Figures 3-8 represent stereo-pair scatter plots. The origin was chosen to represent the power $46 \mathbf{I}+0 \mathbf{J}$ -2 K D. Each stereo-pair scatter plot includes a 95\% ellipsoidal surface of constant probability density (distribution ellipsoid) which provides an indication of the spread of the sample based on the ellipsoid's size, shape and orientation. Figures 3 and 4 show the stereo-pairs representing the keratometric measurements taken prior to contact lens wear (S1-sample 1) and after three hours of contact lens wear (S2- sample 2) respectively.

Figure 5 is a representation of Figures 3 and 4 superimposed. It is clearly evident from Figures 3 to 5 that there is more variation before contact lens wear (red data points) than after three hours of contact lens wear (blue data points). This variation occurs predominantly along the stigmatic axis and can be verified by the quantitative statistics represented in Table 2. The three possible outliers in Figure 3 and the one possible outlier in Figure 2 seen outside the ellipsoid (points which the investigators assumed as irregularities in the sample) may have influenced the variation observed. When the stereo-pairs in Figure 3 are fused, one will notice that the spread of the majority of the data points do not coincide with the orientation of the ellipsoid. Therefore it was decided to remove all possible outliers from both samples to see what effect the possible outliers have on the results (Figures 6 to 8 and Table 3). When comparing Figures 3 and 6, an obvious change in size, shape and orientation of the distribution ellipsoid can be seen. However, there is a less dramatic change induced in the second sample with the removal of possible outliers as is evident when comparing Figures 4 and 7. Figure 8 provides a representation of Figures 6 and 7 superimposed. Hy- 
pothesis testing ${ }^{19}$ of means and variance-covariance matrices (Table 4) was done on the data both with and without possible outliers. These tests were done at a $95 \%$ confidence level. The null hypothesis was rejected if the test statistic was greater than the critical value. According to the hypothesis testing there was statistically significant change in keratometric behaviour for means (test statistic: 18.95 for sample including possible outliers and 31.18 for sample devoid of possible outliers) and variances (test statistic: 122.65 for sample including possible outliers and 43.36 for sample devoid of possible outliers) after contact lens wear even when possible outliers were removed. Table 4 provides further information on the hypothesis tests.

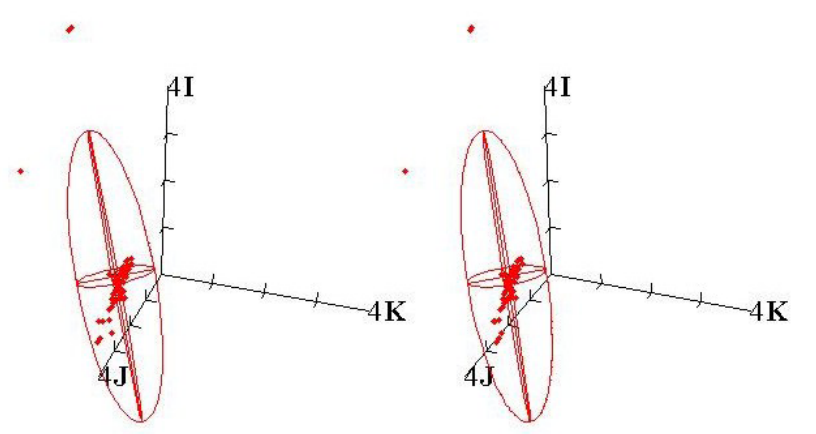

Figure 3. Stereo-pair scatter plot (with included 95\% distribution ellipsoid) of measurements taken before contact lens wear. Figures 3 to 8 all have a tick interval of $\mathbf{I}, 1 \mathbf{J}, 1 \mathbf{K}$ and origin at $46 \mathbf{I}+0 J-2 \mathbf{K}$.
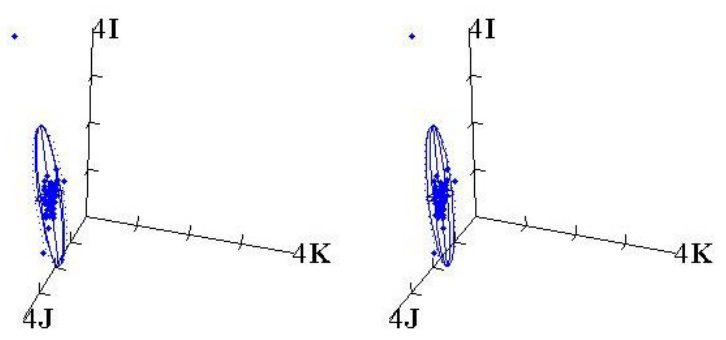

Figure 4. Stereo- pair scatter plot (with included 95\% distribution ellipsoid) of measurements taken three hours after contact lens wear.

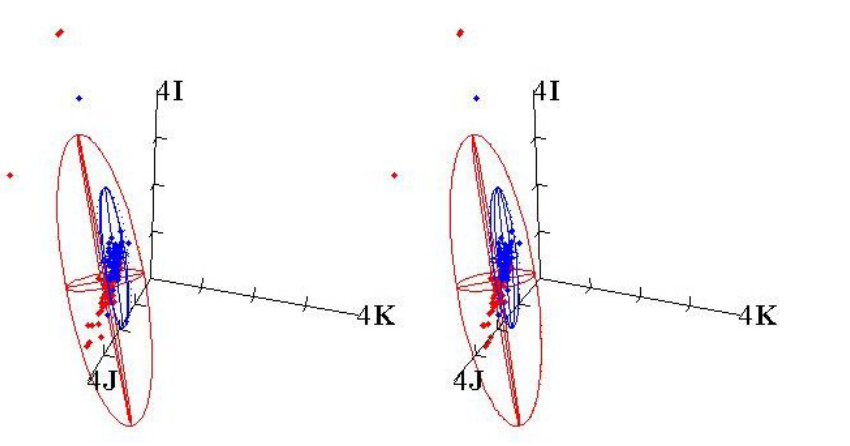

Figure 5. Stereo- pair scatter plot representing the superimposed image of Figures 3 and 4.
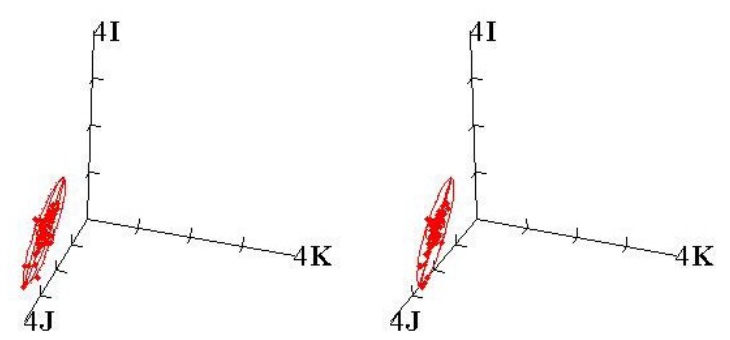

Figure 6. Stereo- pair scatter plot (with included 95\% distribution ellipsoid) of measurements taken before three hours of contact lens wear with possible outliers removed.
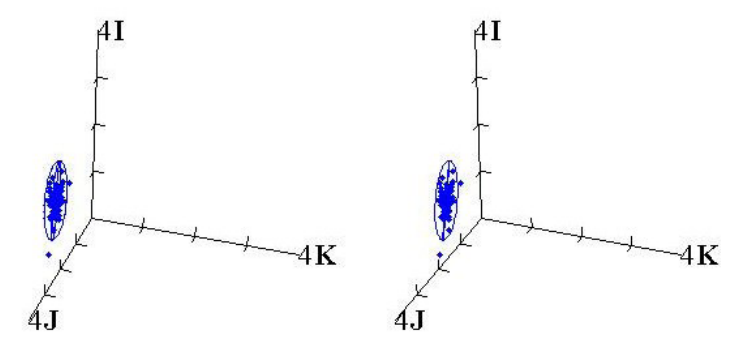

Figure 7. Stereo- pair scatter plot (with included 95\% distribution ellipsoid) of measurements taken three hours after contact lens wear with possible outliers removed. 

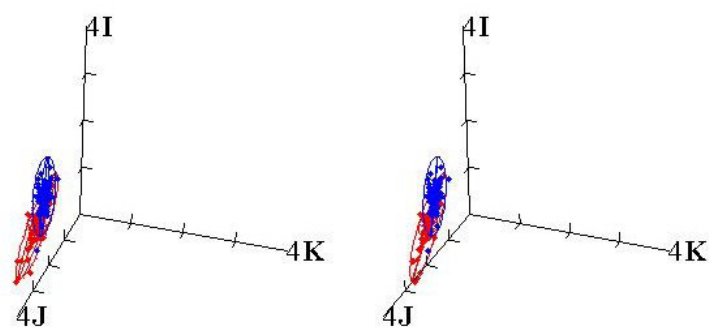

Figure 8. Stereo- pair scatter plot representing the superimposed image of Figures 6 and 7.

Table 2. Statistics (mean powers in conventional and scientific notation, variance-covariance matrices and volume) for auto-keratometric measurements taken before three hours of contact lens wear (S1) and after three hours of contact lens wear (S2). These samples include possible outliers.

\begin{tabular}{|c|c|c|}
\hline & S1 (before) & S2 (after) \\
\hline Conventional mean & $48.75 /-5.62 \times 138$ & $49.03 /-5.37 \times 136$ \\
\hline $\begin{array}{l}\text { Scientific notation } \\
\text { (D) }\end{array}$ & $45.94 \mathbf{I}+0.26 \mathbf{J}-2.80 \mathrm{~K}$ & $46.35 \mathrm{I}+0.08 \mathrm{~J}-2.68 \mathrm{~K}$ \\
\hline $\begin{array}{l}\text { Variance-covariance } \\
\left(D^{2}\right)\end{array}$ & $\mathbf{S}=\left(\begin{array}{ccc}0.596 & -0.353 & -0.252 \\
-0.353 & 0.348 & 0.189 \\
-0.252 & 0.189 & 0.198\end{array}\right)$ & $\mathbf{S}=\left(\begin{array}{ccc}0.137 & -0.088 & -0.049 \\
-0.088 & 0.083 & 0.046 \\
-0.049 & 0.046 & 0.036\end{array}\right)$ \\
\hline $\begin{array}{l}\text { Volume of } 95 \% \\
\text { distribution ellipsoid }\left(\mathrm{D}^{3}\right)\end{array}$ & 2.11 & 1.53 \\
\hline
\end{tabular}

Table 3. Statistics (mean powers in conventional and scientific notation, variance-covariance matrices and volume) for auto-keratometric measurements taken before three hours of contact lens wear (S1) and after three hours of contact lens wear (S2). These statistics represent the samples devoid of the possible outliers.

\begin{tabular}{|c|c|c|}
\hline & S1 (before) & S2 (after) \\
\hline Conventional mean & $48.51 /-5.44 \times 139$ & $48.97 /-5.33 \times 136$ \\
\hline $\begin{array}{l}\text { Scientific notation } \\
\text { (D) }\end{array}$ & $45.79 \mathbf{I}+0.36 \mathbf{J}-2.70 \mathbf{K}$ & $46.31 \mathrm{I}+0.11 \mathrm{~J}-2.66 \mathrm{~K}$ \\
\hline $\begin{array}{l}\text { Variance-covariance } \\
\left(D^{2}\right)\end{array}$ & $\mathbf{S}=\left[\begin{array}{rrr}0.144 & -0.025 & 0.036 \\
-0.025 & 0.043 & 0.004 \\
0.036 & 0.004 & 0.016\end{array}\right]$ & $\mathbf{S}=\left[\begin{array}{ccc}0.060 & -0.021 & -0.002 \\
-0.021 & 0.024 & 0.005 \\
-0.002 & 0.005 & 0.007\end{array}\right]$ \\
\hline $\begin{array}{l}\text { Volume of } 95 \% \\
\text { distribution ellipsoid } \\
\left(D^{3}\right)\end{array}$ & 1.33 & 0.63 \\
\hline
\end{tabular}


Table 4. Results for hypothesis testing on the means and variance-covariance matrices of S1 and S2 with and without possible outliers.

\begin{tabular}{|c|c|c|c|}
\hline MEANS & Critical value & Test statistic & Statistically different \\
\hline $\begin{array}{l}\text { Samples including } \\
\text { possible outliers }\end{array}$ & $F_{(\alpha, 3,116)} \approx 2.68$ & 18.9458 & Yes \\
\hline $\begin{array}{l}\text { Samples devoid of } \\
\text { possible outliers }\end{array}$ & $F_{(\alpha, 3,112)} \approx 2.68$ & 31.1787 & Yes \\
\hline \multicolumn{4}{|l|}{$\begin{array}{l}\text { VARIANCE- } \\
\text { COVARIANCE }\end{array}$} \\
\hline $\begin{array}{l}\text { Samples including } \\
\text { possible outliers }\end{array}$ & $\chi^{2}(\alpha, \sigma)=12.592$ & 122.6512 & Yes \\
\hline $\begin{array}{l}\text { Samples devoid of } \\
\text { possible outliers }\end{array}$ & $\chi_{(\alpha, \sigma)}^{2}=12.592$ & 43.3576 & Yes \\
\hline
\end{tabular}

\section{Discussion}

Variations in keratometric data can always be expected when conducting studies that include measurements taken on human subjects $7,20,21$. This could be due to a plethora of reasons some of which include fluctuations in the tear layer and other ocular components, eye movements, blinking and clinician-instrument interaction. This is what makes the interpretation of such studies challenging. In an attempt to provide the most complete and accurate analysis possible, samples with and without possible outliers will be discussed.

On inspection of Figures 3-5 it is obvious that there is more keratometric variation present before contact lens wear (suggesting that rigid contact lens wear might have provided some sort of stability in that keratoconic cornea). This is further substantiated by the quantitative statistics presented in Table 2. For a deeper understanding of the quantitative statistics presented in this paper, refer to publications by Harris ${ }^{15}$, 16, 19, 22, 23. Hypothesis testing also verified that there was a statistically significant difference in keratometric behaviour after contact lens wear. However, when comparing the means of S1 and S2, the change in keratometric behaviour does not appear to be clinically significant. Overall, the most change occurred along the stigmatic axis, however, there were also changes that occurred along the ortho-antistigmatic and oblique-antistigmatic axes as well (refer to the variance-covariance of S1 and S2 in Table 2).

After possible outliers were removed from $\mathrm{S} 1$ and S2 the keratometric variation decreased. There was a dramatic change in the size, shape and direction of the ellipsoid representing S1 but less of a change in the ellipsoid representing S2 (compare Figure 3 with 6 and Figure 4 with 7). With the possible outliers removed, the spread of the data points coincides with the orientation of the ellipsoid for both samples (Figures 6 and 7). Even though the keratometric variation was reduced with the removal of the possible outliers, the hypothesis testing still showed that contact lens wear statistically significantly influenced keratometric behaviour and Table 3 provides further support for this finding. However, the three hours of contact lens wear had little clinically significant affect on the keratometric behaviour.

As this was a pilot study, one would expect a few shortcomings. It is unlikely that a three hour period would allow for diurnal changes to make a significant impact on keratometric behaviour, however, there was no control group therefore diurnal effects may need to be considered. Other limitations of the study include an inadequate number of subjects, measurements taken before and after a three hour period only instead of at regular intervals throughout the day and 
measurements were done on a mildly keratoconic cornea only.

\section{Conclusion}

So what exactly does this all mean? The introduction of a PMMA rigid contact lens onto a keratoconic cornea appears to reduce variation in keratometric behaviour (possibly bringing about some sort of stability to the keratoconic cornea). However, it is still unclear if all of the variation observed can be totally attributed to the presence of the contact lens. Rigid contact lens wear also seemed to cause a stigmatic increase in corneal curvature which was statistically significant but perhaps not necessarily clinically significant (approximately $0.25 \mathrm{D}$ difference in the spherical component of the conventional mean). Apart from the obvious stigmatic increase in corneal curvature, there were also changes that occurred along the ortho-antistigmatic and oblique-antistigmatic axes as well (refer to the variance-covariance of S1 and S2 in Table 2). The multivariate statistical methods used in this study provide a sound foundation for further investigations that are necessary in this area of research so that a better understanding of the keratometric behaviour of the keratoconic cornea can be achieved. The first investigator of this study is currently involved with further studies investigating the effect of rigid contact lenses on keratometric behaviour in normal corneas.

\section{References}

1. Rubin A. Short-term variation of refractive behaviour in human eyes. Masters degree dissertation, Rand Afrikaans University, Johannesburg, South Africa, 1993.

2. Rengstorff RH. The Fort Dix report- a longitudinal study of the effects of contact lenses. Am J Optom Arch Am Acad Optom 196542 753-763.

3. Rengstorff RH. Corneal curvature and astigmatic changes subsequent to contact lens wear. J Am Optom Assoc 1965 36 996-1000.

4. Rengstorff RH. Variations in corneal curvature measurements: an after effect observed with habitual wearers of contact lenses. Am J Optom Arch Am Acad Optom 1969 46 45-51.

5. Rengstorff RH. Changes in corneal curvature associated with contact lens wear. J Am Optom Assoc 197950375 376.

6. Misage RJ. Changes in corneal curvature with contact lens wear. J Am Optom Assoc 196233 590-592.

7. Gillan WDH. Corneal topographical change induced by rigid PMMA contact lenses. S Afr Optom 200362 166172.
8. Davis LJ, Schechtman KB, Wilson BS, Rosenstiel CE, Riley CH, Libassi DP, Gundel RE, Rosenberg L, Gordon MO, Zadnik K. Longitudinal changes in visual acuity in keratoconus. Inv Ophthalmol Vis Sci 200647 489-500.

9. Wagner H, Barr JT, Zadnik K. Collaborative Longitudinal Evaluation of Keratoconus (CLEK) Study: Methods and findings to date. Contact Lens Ant Eye 200730 223-232.

10. Rabinowitz YS. Keratoconus. Surv Ophthalmol. 199842 297-319.

11. Cronje-Dunn S. Short-term keratometric variation in the human eye. Masters degree dissertation, Rand Afrikaans University, Johannesburg, South Africa, 1995.

12. Hannush SB, Crawford SL, Waring G, Gemmill MC, Lynn MJ, Nizam A. Accuracy and precision of keratometry, photokeratoscopy, and corneal modelling on calibrated steel balls. Arch Ophthalmol 1989107 1235-1239.

13. Mathebula SD, Rubin A. A comparative study of autokeratometric and Scheimpflug keratometric measurements of the anterior corneal surface: results for a single subject. S Afr Optom 200766 109-119.

14. Du Toit I. The effect of physical exercise on keratometric variation in the human eye. Masters degree dissertation, Rand Afrikaans University, Johannesburg, South Africa, 2001.

15. Harris WF. Graphical representation of symmetric dioptric powers and the concept of stigmatic, astigmatic and antistigmatic power. S Afr Optom 200261 126-134.

16. Harris WF. Representation of dioptric power in Euclidian 3-space. Ophthal Physiol Opt 199111 130-136.

17. Malan DJ. Dioptric power data analysis. S Afr Optom 1993 52 84-90.

18. Malan DJ. The excess of objective automatic refraction over subjective clinical refraction: methods of analysis, results. Masters degree dissertation, Rand Afrikaans University, Johannesburg, South Africa, 1994.

19. Harris WF. Testing hypotheses on dioptric power. Optom Vis Sci 199269 835-845.

20. Cronje S, Harris WF. Short-term keratometric variation in the human eye. Optom Vis Sci 199774 420-424.

21. Gillan WDH. Variation in surface power and thickness of a moderately keratoconic cornea. S Afr Optom 200867 410.

22. Harris WF. The matrix representation of dioptric power. Part 1: an introduction. S Afr Optom 198847 19-23.

23. Harris WF. Dioptric power: its nature and its representation in three- and four dimensional space. Optom Vis Sci 199774 349-366. 
